\title{
Collocation Solutions of a Weakly Singular Volterra Integral Equation
}

T. DIOGO ${ }^{1}$, P. LIMA ${ }^{2}$, Centro de Matemática e Aplicações, Instituto Superior Técnico, Av. Rovisco Pais, 1049-001 Lisboa, Portugal.

\begin{abstract}
The discrete superconvergence properties of spline collocation solutions for a certain Volterra integral equation with weakly singular kernel are analyzed. In particular, the attainable convergence orders at the collocation points are examined for certain choices of the collocation parameters.
\end{abstract}

\section{Introduction}

The Volterra integral equation

$$
\begin{gathered}
y(t)=\int_{0}^{t} K(t, s) p(t, s) y(s) d s+g(t), \quad t \in I=(0, T], \\
p(t, s):=\frac{s^{\mu-1}}{t^{\mu}}
\end{gathered}
$$

where $\mu>0, K(t, s)$ is a smooth function and $g$ is a given function, can arise, e.g., in heat conduction problems with mixed boundary conditions ([2], [10]). The case when $K(t, s)=1$ has been considered in several papers. The following lemma summarizes the analytical results for (1.1) in the case $K(t, s)=1$.

Lemma 1.1. (a) [12] Let $\mu>1$ in (1.2). If the function $g$ belongs to $C^{m}[0, T]$ then the integral equation

$$
y(t)=\int_{0}^{t} p(t, s) y(s) d s+g(t), \quad t \in(0, T]
$$

possesses a unique solution $y \in C^{m}[0, T]$.

(b) [16] In the above case (a), the unique solution $y \in C^{m}[0, T]$ is given by

$$
y(t)=g(t)+t^{1-\mu} \int_{0}^{t} s^{\mu-2} g(s) d s .
$$

(c) [16] However, if $0<\mu \leq 1$ and $g \in C^{1}[0, T]$ (with $g(0)=0$ if $\mu=1$ ), then (1.3) has a family of solutions in $C[0, T]$ of which only one has $C^{1}$ continuity.

\footnotetext{
${ }^{1}$ tdiogo@math.ist.utl.pt

${ }^{2}$ plima@math.ist.utl.pt
} 
We note that equation (1.3) differs substantially from the well known case of Abel-type equations whose kernels contain a singularity of the form $q(t, s)=$ $(t-s)^{-\alpha}, 0<\alpha<1$ (see e.g. [6], [15] for comprehensive studies). For these equations, a smooth forcing function leads to a solution which has typically unbounded derivatives at $t=0$. As a consequence of this non-smooth behaviour, if uniform meshes are used then the convergence order of polynomial spline collocation methods (studied in detail in [6]) is only $1-\alpha$, independently of the degree of the polynomials. In order to recover the optimal convergence orders one has to use suitable graded meshes ([3], [8], [19]). Alternatively, one may keep the uniform meshes but then use nonpolynomial spline approximating functions reflecting the singularity; variable transformations followed by standard methods have also been considered by several authors (e.g. [13], [14], [1], [18]). For an extensive list of references on these and other approaches see [4].

Equation (1.3) has been the subject of several works for the case when $\mu>1$ : certain classes of product integration methods based on Newton-Cotes rules were studied in [10]; Diogo et al [12] considered a fourth order Hermite-type collocation method and Lima and Diogo [17] developed an extrapolation algorithm, based on Euler's method. Recently, it was shown that general collocation methods on uniform meshes based on piecewise polynomials of degree $m-1$ yield convergence of order $m$. In [11] it was also proved that if certain derivatives of the exact solution are zero at the origin, then a higher order is attained at the mesh points by a special choice of the collocation points, like the Radau II points. In this work we investigate the use of the Gauss-Legendre points (that is, the zeros of the shifted Legendre polynomial $\left.P_{m}(2 s-1)\right)$ as collocation parameters. In this case, the interesting feature is that superconvergence does not occur at the mesh points, that is, the superconvergence results for ODEs when using the Gauss points as collocation parameters do not carry over to second kind Volterra equations. However, in Section 3 of this work we give sufficient conditions for superconvergence to be attained at the collocation points.

The above properties are in sharp contrast with the situation for Abel-type equations for which no superconvergence properties can be obtained ([7], [6], [4]).

\section{Collocation Methods}

Let us consider equation (1.3), that is, (1.1) with $K(t, s)=1$, and in future work the results will be extended to a more general function $K$. We shall take $\mu>1$.

We follow the notations of [6]. Given the following partition of the interval $I$

$$
\left\{t_{j}=j h, \quad 0 \leq j \leq N ; N h=T\right\}
$$

let $\sigma_{0}:=\left[t_{0}, t_{1}\right], \sigma_{n}:=\left(t_{n}, t_{n+1}\right], 1 \leq n \leq N-1$ and define $Z_{N}:=\left\{t_{n}: n=\right.$ $1, \ldots, N-1\}, \bar{Z}_{N}:=Z_{N} \cup T$. Furthermore, let $\pi_{m-1}$ denote the space of polynomials of degree $m-1$. The exact solution of (1.3) will be approximated in the piecewise polynomial space

$$
S_{m-1}^{-1}\left(Z_{N}\right):=\left\{u:\left.u\right|_{\sigma_{n}}=: u_{n} \in \pi_{m-1}, \quad 0 \leq n \leq N-1\right\},
$$


whose elements, in general, will possess jump discontinuities at their knots $Z_{N}$. Consider the following finite subset of $I$

$$
X(N)=\bigcup_{n=0}^{N-1} X_{n}
$$

with

$$
X_{n}:=\left\{t_{n j}:=t_{n}+c_{j} h: 0 \leq c_{1}<\ldots<c_{m} \leq 1\right\} .
$$

The approximate solution $u \in S_{m-1}^{-1}\left(Z_{N}\right)$ will be required to satisfy the original equation (1.3) on $X(N)$ (set of collocation points). We thus have the following collocation equation

$$
u(t)=g(t)+\int_{0}^{t} p(t, s) u(s) d s, \quad t \in X(N),
$$

which can be rewritten as

$$
\begin{aligned}
u_{n}\left(t_{n j}\right)=g\left(t_{n j}\right)+ & \int_{0}^{c_{j}} \frac{(n+\tau)^{\mu-1}}{\left(n+c_{j}\right)^{\mu}} u_{n}\left(t_{n}+\tau h\right) d \tau \\
& +\sum_{i=0}^{n-1} \int_{0}^{1} \frac{(i+\tau)^{\mu-1}}{\left(n+c_{j}\right)^{\mu}} u_{i}\left(t_{i}+\tau h\right) d \tau, \\
& j=1, \ldots, m(n=0, \ldots, N-1) .
\end{aligned}
$$

Let $\lambda_{l}$ be the canonical Lagrange polynomials associated with the collocation parameters, defined by

$$
\lambda_{l}(\tau):=\prod_{\substack{i=1 \\ i \neq l}}^{m} \frac{\tau-c_{i}}{c_{l}-c_{i}}
$$

If on each subinterval $\sigma_{n} u$ is given by its Lagrange formula

$$
u_{n}\left(t_{n}+\tau h\right):=\sum_{l=1}^{m} \lambda_{l}(\tau) U_{n l}, \quad t_{n}+\tau h \in \sigma_{n},
$$

where $U_{n l}:=u_{n}\left(t_{n}+c_{l} h\right)$, then (2.5) represents a sequence of $N$ linear systems in the unknowns $\left(U_{n 1}, \ldots, U_{n m}\right)^{T}, 0 \leq n \leq N-1$ :

$$
\begin{aligned}
U_{n j}=g\left(t_{n j}\right) & +\sum_{l=1}^{m}\left(\int_{0}^{c_{j}} \frac{(n+\tau)^{\mu-1}}{\left(n+c_{j}\right)^{\mu}} \lambda_{l}(\tau) d \tau\right) U_{n l} \\
& +\sum_{i=0}^{n-1} \sum_{l=1}^{m}\left(\int_{0}^{1} \frac{(i+\tau)^{\mu-1}}{\left(n+c_{j}\right)^{\mu}} \lambda_{l}(\tau) d \tau\right) U_{i l} .
\end{aligned}
$$

We see that the integrals in (2.8) can be evaluated analytically.

Let $e:=y-u$ and denote its restriction to the subinterval $\sigma_{n}$ by $e_{n}$. We define

$$
\|e\|_{\infty}:=\sup \left\{\left|e_{n}(t)\right|: t \in \sigma_{n}, \quad n=0, \ldots, N-1\right\} .
$$

We have the following global convergence result [9]. 
Theorem 2.1. Suppose $\mu>1$ in (1.2). Let $u \in S_{m-1}^{(-1)}\left(Z_{N}\right)$ denote the collocation approximation to the solution of the integral equation (1.3) and assume that $g \in$ $C^{m}(I)$. Then, for every choice of the collocation parameters $\left\{c_{j}\right\}$, with $0 \leq c_{1}<$ $\ldots<c_{m} \leq 1$, we have

$$
\|e\|_{\infty}=O\left(h^{m}\right), \quad(\text { as } h \downarrow 0, N h=T) .
$$

\section{Superconvergence}

In [11] we investigated the discrete superconvergence properties of the collocation solutions for equation (1.3). By this we mean the possibility of attaining a higher order $p>m$ at the mesh points, by a special choice of the collocation points. We showed that if certain derivatives of $y$ vanish at the origin then a higher order of the collocation error near the origin is attained. This fact together with a judicious choice of the collocation points leads to discrete superconvergence at the mesh points. In particular, we obtained the following result.

Theorem 3.1. Consider $\mu>1$ in (1.3) and let $u \in S_{m-1}^{(-1)}\left(Z_{N}\right)$ denote the collocation approximation to the solution of the integral equation (1.3). Let the collocation parameters $\left\{c_{j}\right\}$ be the Radau II points for $(0,1]$, that is, the zeros of $P_{m-1}(2 s-1)-P_{m}(2 s-1)$, where $P_{m}$ is the $m^{\text {th }}$ degree Legendre polynomial. If $g \in C^{2 m-1}[0, T]$ and

$$
y^{(m)}(0)=y^{(m+1)}(0)=\cdots=y^{(2 m-2)}(0)=0, \quad m \geq 1
$$

then we have local superconvergence of order $2 m-1$ at the mesh points, that is,

$$
\max _{t_{n} \in \bar{Z}_{N}}\left|e\left(t_{n}\right)\right|=O\left(h^{2 m-1}\right), \quad(\text { as } h \downarrow 0, N h=T) .
$$

However, if we take as collocation parameters the Gauss-Legendre points (that is, the zeros of the shifted Legendre polynomial $\left.P_{m}(2 s-1)\right)$ then superconvergence does not occur at the mesh points.

On the other hand, we now show that the Gauss points lead to superconvergence at the collocation points which is stated in the following theorem.

Theorem 3.2. Let $\mu>1$ and $u \in S_{m-1}^{(-1)}\left(Z_{N}\right)$ denote the collocation approximation to the solution of the integral equation (1.3), defined by (2.4), where the collocation parameters $\left\{c_{j}\right\}$ are the Gauss points for $(0,1)$ and let $g \in C^{m+1}[0, T]$. If the $m$-th order derivative of $y$ is such that

$$
y^{(m)}(0)=0, \quad m \geq 1
$$

then we have superconvergence of order $m+1$ at the collocation points, that is

$$
\max _{t_{n j} \in X_{N}}\left|e\left(t_{n j}\right)\right|=O\left(h^{m+1}\right), \quad(\text { as } h \downarrow 0, N h=T) .
$$


Proof. It will be convenient to rewrite the collocation equation (2.4) in the form

$$
u(t)=g(t)+\int_{0}^{t} p(t, s) u(s) d s-\delta(t), \quad t \in I,
$$

where $\delta$ is a suitable function which is zero at the collocation points $\delta\left(t_{n j}\right)$. Moreover $\delta$ will be smooth on each subinterval, with the degree of smoothness given by that of $g$. Subtracting (3.5) from (1.3) gives the following second kind Volterra integral equation for the error function

$$
e(t)=\delta(t)+\int_{0}^{t} p(t, s) e(s) d s, \quad t \in I,
$$

which is of the type of (1.3) and whose solution is given by (cf. (1.4))

$$
e(t)=\delta(t)+\int_{0}^{t} \frac{s^{\mu-2}}{t^{\mu-1}} \delta(s) d s, \quad t \in I .
$$

We now analyze (3.7) at $t=t_{n j}$, using the fact that $\delta\left(t_{n j}\right)=0$. In the case $t=t_{0 j}$, since $y \in C^{m+1}[0, T]$ and $y^{(m)}(0)=0$, it can be proved that (cf. [11])

$$
\max _{1 \leq j \leq m}\left|e\left(t_{0 j}\right)\right|=O\left(h^{m+1}\right) .
$$

Consider now the case when $t=t_{n j}, n \geq 1$. By making appropriate changes of variables in (3.7) yields

$$
\begin{aligned}
e\left(t_{n j}\right)= & \sum_{i=0}^{n-1} h \int_{0}^{1} \frac{\left(t_{i}+\tau h\right)^{\mu-2}}{\left(t_{n j}\right)^{\mu-1}} \delta\left(t_{i}+\tau h\right) d \tau \\
& +h \int_{0}^{c_{j}} \frac{\left(t_{n}+\tau h\right)^{\mu-2}}{\left(t_{n j}\right)^{\mu-1}} \delta\left(t_{n}+\tau h\right) d \tau .
\end{aligned}
$$

If $i=0$, we have

$$
\int_{0}^{1} \frac{(\tau h)^{\mu-2}}{\left(t_{n}\right)^{\mu-1}} \delta(\tau h) d \tau=\int_{0}^{1}\left(\frac{\tau h}{t_{n}}\right)^{\mu-1} \frac{\delta(\tau h)}{\tau h} d \tau \leq \int_{0}^{1} \frac{\delta(\tau h)}{\tau h} d \tau,
$$

where we have used the fact that $\left(\tau h / t_{n}\right)^{\mu-1} \leq 1$. By a similar analysis as in [11] it can be shown that

$$
\int_{0}^{1} \frac{\delta(\tau h)}{\tau h} d \tau=O\left(h^{m}\right)
$$

If $i \geq 1$ we proceed as follows:

$\int_{0}^{1} \frac{\left(t_{i}+\tau h\right)^{\mu-2}}{\left(t_{n j}\right)^{\mu-1}} \delta\left(t_{i}+\tau h\right) d \tau=\int_{0}^{1} \frac{\left(t_{i}+\tau h\right)^{\mu-1}}{\left(t_{n j}\right)^{\mu-1}} \frac{1}{\left(t_{i}+\tau h\right)} \delta\left(t_{i}+\tau h\right) d \tau \leq \int_{0}^{1} \frac{\delta\left(t_{i}+\tau h\right)}{t_{i}+\tau h} d \tau$

Using an $m$-point quadrature rule based on the abscissas $t_{i l}$, with remainder $E_{n i}$, for each integral, we obtain

$$
\int_{0}^{1} \frac{\delta\left(t_{i}+\tau h\right)}{t_{i}+\tau h} d \tau=\sum_{l=1}^{m} b_{l} \frac{\delta\left(t_{i l}\right)}{t_{i l}}+E_{n i}=O\left(h^{m+1}\right)
$$


where we have used the fact that $\delta\left(t_{i l}\right)=0$; on the other hand, since the $c_{j}$ satisfy the orthogonality condition

$$
\int_{0}^{1} \prod_{j=1}^{m}\left(s-c_{j}\right) d s=0
$$

and the integrand is in the class $C^{m+1}$, then $E_{n i}=O\left(h^{m+1}\right)$ (see e.g. [5]).

Finally, for the last integral in (3.9), we have

$$
h \int_{0}^{c_{j}} \frac{\left(t_{n}+\tau h\right)^{\mu-2}}{\left(t_{n j}\right)^{\mu-1}} \delta\left(t_{n}+\tau h\right) d \tau \leq h \int_{0}^{c_{j}} \frac{\delta\left(t_{n}+\tau h\right)}{t_{n}+\tau h} d \tau .
$$

From (3.6) we can easily obtain the relation

$$
|\delta(t)| \leq \frac{\mu+1}{\mu} \sup _{t \in\left(t_{n}, t_{n j}\right]}|e(t)|, \quad t \in\left(t_{n}, t_{n j}\right],
$$

which, together with (3.14), gives

$$
h \int_{0}^{c_{j}} \frac{\left(t_{n}+\tau h\right)^{\mu-2}}{\left(t_{n j}\right)^{\mu-1}} \delta\left(t_{n}+\tau h\right) d \tau \leq C^{*} h \int_{0}^{c_{j}} \delta\left(t_{n}+\tau h\right) d \tau=O\left(h^{m+1}\right) .
$$

In the analysis above we have assumed that $t_{n}>h$, since the analysis for $e(t)$, with $t$ near the origin, has been considered in (3.11). Summing up the contributions (3.11), (3.12), (3.16) in (3.9) yields

$$
\begin{aligned}
\left|e\left(t_{n j}\right)\right| & \leq h O\left(h^{m}\right)+h \sum_{i=1}^{n-1}\left|E_{n i}\right|+O\left(h^{m+1}\right) \\
& \leq O\left(h^{m+1}\right), \quad n=1, \ldots, N .
\end{aligned}
$$

From (3.17) and (3.8) we obtain the desired result (3.4).

Remark 1. We note that the result of Theorem 3.2 can be generalized as follows. If the collocation points $t_{i j}=t_{i}+c_{j} h$ are such that the collocation parameters $\left\{c_{j}\right\}$ satisfy the orthogonality condition (3.13), then the result (3.4) remains valid. A further example of such parameters are the Radau II points and a numerical illustration is given in the next section (cf. Table 4).

\section{Numerical Results}

We have considered the numerical solution of equation (1.3), with various choices of $g(t)$ and, consequently, of $y(t)$, for $t \in[0,2]$.

Example 1. $\mu=1.5$ and $g$ such that $y(t)=t^{4}+t^{4.5}$.

Example 2. $\mu=1.5$ and $g$ such that $y(t)=t^{5}+t^{5.4}$.

Example 3. $\mu=1.5$ and $g$ such that $y(t)=t^{3}$. 
Example 4. $\mu=1.5$ and $g$ such that $y(t)=t^{4}$.

Tables 1-5 illustrate the performance of the collocation method defined by (2.8) applied to the above examples.

In Table 1 we have considered collocation in $S_{m-1}^{-1}\left(Z_{N}\right)$, with $m=3$, applied to Example 1. We have used the Gauss points $\left\{c_{1}=(5-\sqrt{15}) / 10, c_{2}=1 / 2, c_{3}=\right.$ $(5+\sqrt{15}) / 10\}$ as collocation parameters and the results of the first column confirm the discrete convergence order $m=3$, that is, no superconvergence at the mesh points. However, since $y^{\prime \prime \prime}(0)=0$, that is, (3.3) is satisfied, local superconvergence of order $m+1=4$ takes place at the collocation points and this is shown in the third column. Table 2 displays the results obtained with cubic collocation, that is, in $S_{m-1}^{-1}\left(Z_{N}\right)$, with $m=4$, applied to Example 2. Again we have used the Gauss points, now given by

$$
\begin{array}{ll}
c_{1}=\frac{35-\sqrt{525+70 \sqrt{30}}}{70}, & c_{2}=\frac{35-\sqrt{525-70 \sqrt{30}}}{70}, \\
c_{3}=\frac{35+\sqrt{525-70 \sqrt{30}}}{70}, & c_{4}=\frac{35+\sqrt{525+70 \sqrt{30}}}{70} .
\end{array}
$$

The results of the first column confirm the discrete convergence order $m=4$ (no superconvergence at the mesh points) and the third column shows local superconvergence of order $m+1=5$ at the collocation points.

Table 1: Quadratic collocation using Gauss points Example 1: $y(t)=t^{4}+t^{4.5}$, $t \in[0,2]$

\begin{tabular}{|l|c|c|c|c|}
\hline$h$ & $\max \left|e\left(t_{i}\right)\right|$ & order & $\max \left|e\left(t_{i j}\right)\right|$ & order \\
\hline 0.05 & $1.6 D-4$ & & $1.9 D-6$ & \\
\hline 0.025 & $2.1 D-5$ & 3.0 & $1.1 D-7$ & 4.1 \\
\hline 0.0125 & $2.6 D-6$ & 3.0 & $6.7 D-9$ & 4.1 \\
\hline 0.00625 & $3.2 D-7$ & 3.0 & $4.1 D-10$ & 4.0 \\
\hline 0.003125 & $4.0 D-8$ & 3.0 & $2.5 D-11$ & 4.0 \\
\hline
\end{tabular}

Table 2: Cubic collocation using Gauss points Example 2: $\quad y(t)=t^{5}+t^{5.4}, t \in[0,2]$

\begin{tabular}{|l|c|c|c|c|}
\hline$h$ & $\max \left|e\left(t_{i}\right)\right|$ & order & $\max \left|e\left(t_{i j}\right)\right|$ & order \\
\hline 0.05 & $2.7 D-6$ & & $1.3 D-8$ & \\
\hline 0.025 & $1.7 D-7$ & 4.0 & $3.7 D-10$ & 5.1 \\
\hline 0.0125 & $1.1 D-8$ & 4.0 & $1.1 D-11$ & 5.1 \\
\hline 0.00625 & $6.8 D-10$ & 4.0 & $3.3 D-13$ & 5.1 \\
\hline 0.003125 & $4.3 D-11$ & 4.0 & $1.0 D-14$ & 5.0 \\
\hline
\end{tabular}


For completeness, we use Example 2 to illustrate the result of Theorem 3.1 in the case of quadratic collocation. Since $y^{\prime \prime \prime}(0)=y^{i v}(0)=0$, that is, (3.1) is satisfied, local superconvergence of order $2 m-1=5$ takes place at the mesh points, using the Radau II points $\left\{c_{1}=(4-\sqrt{6}) / 10, c_{2}=(4+\sqrt{6}) / 10, c_{3}=1\right\}$ as collocation parameters. This is shown in the first column of Table 3. The third column displays the errors at the collocation points indicating convergence of order $m+1=4$, in accordance with Remark 1.

In Tables 4, 5 we illustrate the importance of conditions (3.1), (3.3) for attaining superconvergence. The first column of Table 4 shows the errors for quadratic collocation using the Radau II points, applied to Example 3, indicating third order of convergence at the mesh points; we do not have superconvergence and this is not surprising since $y^{\prime \prime \prime}(0) \neq 0$ (cf. (3.1)). The third column of Table 4 shows a similar situation with collocation based on the Gauss points, since (3.3) is not satisfied; there we only have order 3 at the collocation points instead of order $m+1=4$ (cf. $(3.4))$.

Table 5 contains the results for Example 4, obtained with cubic collocation based on the Radau II points (first column) and on the Gauss points (third column). Here, the function $y$ of Example 4 is such that $y^{i v}(0) \neq 0$, therefore conditions (3.1) and (3.3) are not satisfied. Convergence of order 4 is obtained with the Radau II points (on the mesh) and with the Gauss points (at the collocation points), instead of the orders $2 m-1=7$ and $m+1=5$, respectively (cf. (3.2) and (3.4)).

Table 3: Quadratic collocation using Radau II points Example 2: $y(t)=t^{5}+t^{5.4}$, $t \in[0,2]$

\begin{tabular}{|l|c|c|c|c|}
\hline$h$ & $\max \left|e\left(t_{i}\right)\right|$ & order & $\max \left|e\left(t_{i j}\right)\right|$ & order \\
\hline 0.05 & $2.8 D-8$ & & $2.4 D-6$ & \\
\hline 0.025 & $8.1 D-10$ & 5.1 & $1.5 D-7$ & 4.0 \\
\hline 0.0125 & $2.4 D-11$ & 5.1 & $9.2 D-9$ & 4.0 \\
\hline 0.00625 & $7.3 D-13$ & 5.1 & $5.7 D-10$ & 4.0 \\
\hline 0.003125 & $2.2 D-14$ & 5.0 & $3.6 D-11$ & 4.0 \\
\hline
\end{tabular}

Table 4: Quadratic collocation. The importance of conditions (3.1), (3.3). $\max \left|e\left(t_{i}\right)\right|$ and $\max \left|e\left(t_{i j}\right)\right|$ for Example 3

\begin{tabular}{|l|c|c|c|c|}
\hline$h$ & Radau II & $p$ & Gauss & $p$ \\
\hline 0.05 & $3.3 D-6$ & & $6.0 D-6$ & \\
\hline 0.025 & $4.2 D-7$ & 3.0 & $7.5 D-7$ & 3.0 \\
\hline 0.0125 & $5.2 D-8$ & 3.0 & $9.4 D-8$ & 3.0 \\
\hline 0.00625 & $6.5 D-9$ & 3.0 & $1.2 D-8$ & 3.0 \\
\hline 0.003125 & $8.1 D-10$ & 3.0 & $1.5 D-9$ & 3.0 \\
\hline
\end{tabular}


Table 5: Cubic collocation. The importance of conditions (3.1), (3.3). $\max \left|e\left(t_{i}\right)\right|$ and $\max \left|e\left(t_{i j}\right)\right|$ for Example 4

\begin{tabular}{|l|c|c|c|c|}
\hline$h$ & Radau II & $p$ & Gauss & $p$ \\
\hline 0.05 & $3.7 D-8$ & & $9.0 D-8$ & \\
\hline 0.025 & $2.3 D-9$ & 4.0 & $5.6 D-9$ & 4.0 \\
\hline 0.0125 & $1.4 D-10$ & 4.0 & $3.5 D-10$ & 4.0 \\
\hline 0.00625 & $9.1 D-12$ & 4.0 & $4.0 D-11$ & 4.0 \\
\hline 0.003125 & $5.7 D-13$ & 4.0 & $1.4 D-12$ & 4.0 \\
\hline
\end{tabular}

\section{References}

[1] P. Baratella, A.P. Orsi, A new approach to the numerical solution of weakly singular volterra integral equations, J. Comput. Appl. Math., 163 (2004), 401418.

[2] M.A. Bartoshevich, On a heat conduction problem, In ̌.- Fiz. Z̆., 28 (1975), 340-346. (In russian)

[3] H. Brunner, The numerical solution of weakly singular Volterra integral equations by collocation on graded meshes, Math. Comp., 45 (1985), 417-437.

[4] H. Brunner, "Collocation methods for Volterra integral and related functional differential equations", Cambridge University Press, 2004.

[5] H. Brunner, On discrete superconvergence properties of spline collocation methods for nonlinear Volterra integral equations, J. Comput. Appl. Math., 10 (1992), 348-357.

[6] H. Brunner, P.J. van der Houwen, "The Numerical Solution of Volterra Equations", North Holland, 1986.

[7] H. Brunner, S. Nørsett, Superconvergence of collocation methods for Volterra and Abel integral equations of the second kind, Numer. Math., 36 (1981), 347-358.

[8] H. Brunner, A. Pedas, G. Vainikko, The piecewise polynomial collocation method for nonlinear weakly singular Volterra equations, Math. Comp., 68 (1999), 1079-1095.

[9] T. Diogo, A note on collocation and iterated collocation methods for a class of weakly singular Volterra integral equations, submitted.

[10] T. Diogo, N.B. Franco, P. Lima, High order product integration methods for a Volterra integral equation with logarithmic singular kernel, Commun. Pure Appl. Anal., 3 (2004), 217-235. 
[11] T. Diogo, P. Lima, Superconvergence properties of collocation methods for a Volterra integral equation with weakly singular kernel, to appear in J. Comput. Appl. Math..

[12] T. Diogo, S. McKee, T. Tang, A Hermite-type collocation method for the solution of an integral equation with a certain weakly singular kernel, IMA J. Numer. Anal., 11 (1991), 595-605.

[13] T. Diogo, S. Mckee, T. Tang, Collocation methods for second-kind Volterra integral equations with weakly singular kernels, Proc. Roy. Soc. Edinburgh, 124A (1994), 199-210.

[14] E.A. Galperin, E.J. Kansa, A. Makroglou, S.A. Nelson, Variable transformations in the numerical solution of the second kind Volterra integral equations with continous and weakly singular kernels; extension to Fredholm integral equations, J. Comput. Appl. Math., 115 (2000), 193-211.

[15] R. Gorenflo, S. Vessella, "Abel Integral Equations, a Historico-bibliographical Survey", IAGA, Firenze, 1984.

[16] W. Han, Existence, uniqueness and smoothness results for second-kind Volterra equations with weakly singular kernels, J. Integral Equations Appl., 6 (1994), 365-384.

[17] P.M. Lima, T. Diogo, An extrapolation method for a Volterra integral equation with weakly singular kernel, Appl. Numer. Math. 24 (1997), 131-148.

[18] A. Pedas, G. Vainikko, Smoothing transformation and piecewise polynomial collocation for weakly singular Volterra integral equations, Computing, $\mathbf{7 3}$ (2004), 271-293.

[19] T. Tang, A note on collocation methods for Volterra integro-differential equations with weakly singular kernels, IMA J. Numer. Math., 13 (1993), 93-99. 